\title{
Proposta de uma nova metodologia para análise do impacto ambiental do atropelamento de fauna
}

As rodovias representam avanços sociais de grande importância. No entanto também estão associadas a importantes impactos ambientais negativos, sobretudo desmatamento e redução da fauna. Dentre tais efeitos negativos, a mortalidade da fauna por atropelamento tem destaque. O entendimento do impacto ambiental causado pelo atropelamento da fauna é fundamental para o gerenciamento ambiental de rodovias. Até o momento, a avaliação deste tipo de impactos é feita de forma quantitativa e muito superficial. O propósito deste trabalho é propor uma metodologia de avaliação da significância do impacto causado pelo atropelamento da fauna silvestre, através da definição de atributos intrínsecos aos animais silvestres atropelados em função de suas características ecológicas, para em seguida combiná-los segundo uma função matemática predeterminada, aportando peso ao atropelamento de diferentes espécies.

Palavras-chave: Ecologia de estrada; Atropelamento de fauna; Avaliação de impacto ambiental.

\section{Proposal of a new methodology for the analysis of the environmental impact of fauna trampling}

Highways represent major social advances. However, they are also associated with important negative environmental impacts, especially deforestation and fauna reduction. Among such negative effects, fauna mortality by trampling stands out. Understanding the environmental impact caused by fauna trampling is fundamental to the environmental management of highways. So far, the assessment of such impacts is quantitative and very superficial. The purpose of this paper is to propose a methodology for assessing the significance of the impact caused by the trampling of wildlife, by defining intrinsic attributes of wild animals run over according to their ecological characteristics, and then combining them according to a predetermined mathematical function, contributing weight to the trampling of different species.

Keywords: Road ecology; Trampling of fauna; Environmental Impact Assessment.

Topic: Planejamento, Gestão e Políticas Públicas Ambientais

Reviewed anonymously in the process of blind peer.
Received: 15/10/2018

Approved: 16/11/2018
Gustavo Aveiro Lins (iD)

Universidade do Estado do Rio de Janeiro, Brasil

http://lattes.cnpq.br/5173989372426437

http://orcid.org/0000-0002-0244-6925

gustavoaveiro@gmail.com

Oscar Rocha Barbosa

Universidade do Estado do Rio de Janeiro, Brasil

http://lattes.cnpq.br/6551622738384590

http://orcid.org/0000-0001-7838-2393

or-barbosa@hotmail.com

\section{Josimar Ribeiro de Almeida (iD}

Universidade do Estado do Rio de Janeiro, Brasil

http://lattes.cnpq.br/3215586187698472

http://orcid.org/0000-0001-5993-0665

almeida@poli.ufrj.br
Referencing this:

LINS, G. A.; BARBOSA, O. R.; ALMEIDA, J. R.. Proposta de uma nova metodologia para análise do impacto ambiental do atropelamento de fauna. Revista Ibero-Americana de Ciências Ambientais, v.9, n.8, p.273-281, 2018. DOI: http://doi.org/10.6008/CBPC21796858.2018.008.0024 


\section{INTRODUÇÃO}

As estradas, no imaginário popular, estariam associadas à ideia de progresso e modernidade, num "raciocínio de que, quanto mais estradas, melhor, não importando onde ou como" e que até algumas décadas atrás não fazia qualquer menção a nenhum aspecto ambiental dessas construções e sua operação (PRADA, 2004). No entanto, as rodovias também estão associadas a importantes impactos ambientais negativos, sobretudo desmatamento e redução da fauna.

Dentre tais efeitos negativos, a mortalidade da fauna por atropelamento tem destaque (LAURANCE et al., 2014; FAHRIG et al., 2009), isso porque, seu impacto é potencializado por eliminar indivíduos saudáveis das populações (BUJOCZEK et al., 2011), por isso, superando as pressões de predação (BUJOCZEK et al., 2011) e caça (FORMAN et al., 1998), aumentando ainda mais o risco de extinção local dessas espécies (HUCK et al., 2010; JACKSON et al., 2011). Segundo Bager et al. (2012), ocorrem aproximadamente 14,7 milhões de atropelamentos/ano em todo o território brasileiro em suas pouco mais de 1,7 milhões de quilômetros de estradas.

O entendimento do impacto ambiental causado pelo atropelamento da fauna é fundamental, para o gerenciamento ambiental de rodovias, pois permite apoiar a tomada de decisão no que concerne à realização de medidas mitigadoras de maneira mais específica para a situação encontrada, otimizando recursos, a serem feitos em medidas comprovadamente eficazes. Tais características ganham maior relevância, sobretudo no Brasil onde a manutenção da maioria das rodovias está a cargo de órgãos públicos, com orçamento rotineiramente escasso (BAGATINI, 2006).

Até o momento, a maneira usual e a legalmente exigida pelos órgãos ambientais de se avaliar o impacto causado pela rodovia no atropelamento de fauna é simplesmente emitir um relatório que enumera os animais atropelados ou, na melhor das hipóteses, estabelecer uma relação entre a quantidade de animais atropelados por km de rodovia, não levando em conta a espécie, a característica e a função ecossistêmica destes animais atropelados.

O risco de extinção não é análogo a todas as espécies, alguns grupos apresentam maior taxa de extinção do que outros (JOHNSON et al., 2002; JONES et al., 2003). O que sugere que estes grupos compartilham características intrínsecas às espécies que favorecem a situação de extinção (FISHER et al., 2003; CARDILLO et al., 2004).

O objetivo geral deste trabalho é propor uma metodologia de avaliação da significância do impacto causado pelo atropelamento da fauna silvestre em empreendimentos lineares, aportando maior robustez às análises atualmente realizadas. Como objetivo específico é possível destacar a criação de uma metodologia versátil capaz de avaliar o referido impacto em diferentes aspectos da rodovia, como por exemplo, a sua aplicação em momentos distintos da obra de restauração de uma rodovia, para uma análise temporal do impacto, ou em diferentes trechos, objetivando a identificação de trechos mais críticos, ou até mesmo na rodovia como um todo, para posterior comparação com outras rodovias. 


\section{METODOLOGIA}

A metodologia proposta tem como base a correlação entre a frequência de atropelamento e a severidade ecológica da espécie atropelada, através da definição de atributos intrínsecos aos animais silvestres atropelados em função de suas características ecológicas, para em seguida, combiná-los segundo uma função matemática predeterminada, aportando peso ao atropelamento de diferentes espécies.

\section{Cálculo da frequência}

Na metodologia proposta, utilizaremos como medida de frequência a taxa de atropelamentos conforme proposto por BAGER et al. (2012) no livro Ecologia de Estradas. Este modelo atualmente é muito utilizado para representar quantitativamente os atropelamentos dos vertebrados. A possibilidade de poder ser utilizado, em rodovias de qualquer extensão, seja qual for o esforço de amostragem, certamente representa um grande avanço trazendo vantagens ao uso deste método. O referido modelo é calculado, conforme a equação que versa de Cálculo de indivíduos atropelados por quilometro percorrido e pelos dias de monitoramento, que é $T x=(N / K m) / D$, na qual $T x=$ a taxa de atropelamento de indivíduos $(\mathrm{N} / \mathrm{Km} / \mathrm{dia}) ; \mathrm{N}$ = número total de indivíduos atropelados; $\mathrm{Km}$ = distância da rodovia ou do trecho analisado em $\mathrm{km}$; e $\mathrm{D}=$ número de dias dos monitoramentos.

Nota-se que, a taxa de atropelamento de indivíduos (Tx) deverá ser calculada para cada campanha de observação dos animais atropelados, haja vista, que cada campanha poderá apresentar valores diferentes relativos ao número total de indivíduos atropelados $(\mathrm{N})$, ou a distância da rodovia ou do trecho $(\mathrm{Km})$ ou o número de dias de monitoramento (D).

Após o cálculo da Tx de cada campanha, será feito uma média aritmética de todas as campanhas presentes no período, trecho ou na rodovia analisada. Posteriormente, a Tx média, deverá ser confrontado com a tabela 1, de forma a fornecer uma indicação qualitativa da frequência de atropelamento de animais silvestres relativas ao período, trecho ou rodovia analisada.

Tabela 1: Categorias de Frequência de atropelamento de animais vertebrados.

\begin{tabular}{|l|c|}
\hline \multicolumn{1}{|c|}{ Taxa de atropelamentos MÉDIO (indivíduos/Km/dia) } & Categoria \\
\hline $0 \geq 0,005$ & $\mathrm{~A}$ \\
\hline $0,005 \geq 0,015$ & $\mathrm{~B}$ \\
\hline $0,015 \geq 0,025$ & $\mathrm{C}$ \\
\hline $0,025 \geq 0,035$ & $\mathrm{D}$ \\
\hline $0,035 \geq 0,050$ & $\mathrm{E}$ \\
\hline $0,050 \geq 0,1$ & $\mathrm{~F}$ \\
\hline $0,1 \geq 0,3$ & $\mathrm{G}$ \\
\hline 0,3 em diante & $\mathrm{H}$ \\
\hline
\end{tabular}

\section{Cálculo da severidade}

Após a obtenção da frequência de atropelamento, será necessário o cálculo da severidade. 0 objetivo desta etapa é conferir diferentes pesos para o atropelamento de espécies distintas, como por exemplo: o atropelamento de um animal em vias de extinção contribui para redução da biodiversidade local de forma diferente do que o atropelamento de um animal que ainda se encontra em abundância no meio ambiente. 
Considerando a importância do atributo escolhido, bem como a facilidade da sua identificação na população impactada, foram propostos os seguintes atributos para a composição da severidade: Densidade populacional local; Status de conservação da fauna atropelada; Endemismo; e Atropelamento em área de preservação.

\section{Densidade populacional local}

A densidade populacional é um atributo estreitamente relacionado à chance de extinção (EWERS et al., 2006; MACE et al., 2008). Logo, o impacto do atropelamento de um animal que se encontra em uma situação de baixa densidade populacional no local, certamente terá um impacto muito maior do que o atropelamento de um animal pertencente a uma grande população local.

A densidade local será obtida através da análise do monitoramento de fauna presente na região. Espera-se que o estudo de impactos ambientais (EIA) atenda a estas condições. O referido monitoramento deve ser realizado, preferencialmente antes da implementação e operação do empreendimento, haja vista que, após esta etapa, há o risco da alteração das populações locais já estar em andamento; além disso é preciso que a metodologia aplicada esteja em perfeita consonância com a legislação pertinente ao assunto, e que, portanto, esteja homologada pelo órgão ambiental licenciador e, somado a isso, que o monitoramento seja considerado representativo da fauna conhecida para a região, com base em trabalhos científicos ou coleções zoológicas bem como através da aplicação da curva do coletor.

De posse do conhecimento da fauna local, o próximo passo será a classificação das espécies encontradas, para isso, sugerimos a classificação segundo o conceito de constância apresentado por Dajoz (1972), um método já consolidado dentre os estudos ecológicos. Segundo esta metodologia, a constância de captura de uma espécie indica a porcentagem de amostras em que uma determinada espécie esteve presente em um determinado tempo. Esta é determinada da equação do cálculo da constância de ocorrência de cada espécie, que é $F_{A}=P_{A} / P \times 100$, onde $F_{A}$ : Constância; $P_{A}$ : Número de vezes em que a espécie foi registrada nas coletadas; e P: Número total de coletas realizadas. A classificação das espécies quanto à constância é realizada da seguinte forma: Espécie constante - $\geq 50 \%$; Espécie acessória - $25 \% \leq C<50 \%$; e Espécie acidental ou rara - C $<25 \%$.

As espécies atropeladas receberão a mesma classificação (constante, acessória e acidental) que seus análogos receberam em função da sua presença no monitoramento da região. E posteriormente serão pontuados, conforme a tabela 2 . Seguindo a lógica do princípio da precaução, adotaremos o mesmo valor de peso da categoria 'acessória' para os animais não identificados ou não encontrados.

Tabela 2: Peso dos atributos relacionados a densidade populacional local.

\begin{tabular}{|l|c|}
\hline \multicolumn{1}{|c|}{ Classificação } & Peso \\
\hline Constante & 4 \\
\hline Acessória & 6 \\
\hline Acidental & 8 \\
\hline Não encontrado & 6 \\
\hline Não identificado & 6 \\
\hline
\end{tabular}


Por exemplo, se durante o monitoramento fauna local a espécies Didelphis aurita foi classificada como constante, todos os animais da mesma espécie que forem encontrados atropelados receberão o peso 4, correspondente à classificação constante. Desta forma, os animais atropelados que ainda se encontram em relativa abundância no local terão um peso menor do que aqueles considerados raros antes mesmo da implantação do empreendimento.

\section{Status de conservação da fauna atropelada}

Também utilizamos como critério de peso o status de conservação das espécies brasileiras ameaçadas de extinção, através da Lista Oficial das espécies brasileiras ameaçadas de extinção, ou lista nacional, publicada pelo Ministério do Meio Ambiente (MMA), a lista da IUCN (União para a Conservação da Natureza), chamada de lista global. E caso tenha disponível, recomendamos também o uso das listas regionais publicadas pelo órgão estadual e/ou municipal do meio ambiente quando estes existirem.

O sistema desenvolvido pela UICN é a metodologia mais aceita e testada mundialmente. Ela é reconhecidamente objetiva, aplicável para uma ampla variedade de grupos e ambientes, razoavelmente rigorosa e defensável cientificamente e, em geral, produz resultados replicáveis independente do avaliador (VIÉ et al., 2009). A partir da lista de espécies atropeladas, será atribuído uma pontuação conforme preconizado na tabela 3, tendo como base as categorias de extinção presentes nas listas global, nacional e regionais.

Tabela 3: Pontuação de severidade em relação a categorias de extinção.

\begin{tabular}{|c|l|l|}
\hline Severidade & \multicolumn{1}{|c|}{ Sigla } & \multicolumn{1}{c|}{ Categoria } \\
\hline 7 & CR & Criticamente ameaçado (ou criticamente em perigo) \\
\hline 6 & EN & Ameaçado (ou em perigo) \\
\hline 5 & VU & Vulnerável \\
\hline 4 & NT & Quase ameaçado \\
\hline 3 & DD & Dados deficientes \\
\hline 3 & NE & Não avaliado \\
\hline 3 & NA & Não Aplicável \\
\hline 2 & LC & Não ameaçada ou de menor preocupação \\
\hline
\end{tabular}

Tendo em vista que a presença, e, consequentemente, o atropelamento de espécies incluídas nas categorias Extinta (EX), extinto na natureza (EW) e regionalmente extinta (RE), são virtualmente impossíveis, estas categorias foram retiradas da tabela de pontuação de severidade. $O$ emprego de um mesmo sistema com base em diferentes listas para a categorização das espécies fará com que pequenas adaptações sejam necessárias.

Conforme já descrito, não serão raras, as situações nas quais se encontre diferenças nas categorias das espécies em extinção em relação às listas brasileira, global ou regional. Dessa maneira, alguns táxons avaliados nacionalmente, que não correm risco de extinção, podem estar ameaçados de extinção localmente devido a problemas regionais, ou algumas espécies extintas regionalmente, podem ainda ser encontradas em outros estados. 
Em situações em que a lista regional (estadual ou municipal) for utilizada, pode ser necessária uma análise caso a caso, para se estabelecer equivalência entre as categorias de extinção das listas Nacionais (MMA) e internacionais (IUCN) com a regional. Diante desta questão e tendo em vista o princípio da precaução, na metodologia proposta, adotaremos sempre o valor indicado para a classificação mais restritiva.

\section{Endemismo}

As espécies endêmicas são aquelas vulgarmente entendidas como 'não se encontrando em nenhum outro lado', ou seja, são organismos com uma distribuição limitada a habitats especializados, nativos de uma área geográfica restrita, as espécies que ocorrem numa área muito restrita. Devido ao reduzido tamanho de distribuição, as espécies endêmicas estão sempre no limiar da extinção (COLLEN et al., 2006; CARDILLO et al., 2008; FRITZ et al., 2009). Pequenas áreas de distribuição não permitem grandes densidades populacionais, o que pode agir reduzindo a probabilidade de persistência de populações por estocasticidade demográfica, catástrofes locais e endocruzamento, limitando seu potencial de sobrevivência em uma crise de extinção (PURVIS et al., 2000).

As espécies atropeladas serão classificadas como endêmicas ou não endêmicas, tendo como base o Estado do empreendimento. Sua pontuação, terá como base o peso descrito na tabela 4. Novamente em consonância com o princípio da precaução, adotaremos o mesmo valor de peso da categoria 'endêmico' para os animais não identificados.

Tabela 4: Peso dos atributos relacionados a classificação como endêmico.

\begin{tabular}{|l|c|}
\hline \multicolumn{1}{|c|}{ Classificação } & Peso \\
\hline Endêmico & 4 \\
\hline Animal não identificado & 4 \\
\hline Não endêmico & 2 \\
\hline
\end{tabular}

\section{Atropelamento em área de preservação}

Populações de animais silvestres são mais vulneráveis aos impactos das estradas dentro de UCs do que fora de seus limites (PARKS et al., 2002; AMENT et al., 2008). Isso porque, animais silvestres inseridos em um ambiente preservado desempenham plenamente suas funções ecológicas, fundamentais para a manutenção do habitat. Tal entendimento é, inclusive, acolhido na nossa legislação ambiental, mais especificamente na Lei no 9.605 de 12 de fevereiro de 1998 (Lei de Crimes Ambientais), onde no seu Capítulo V, Seção I, art. 29 e $\S$ 4으, diz que 'A pena é aumentada de metade, se o crime é praticado em unidade de conservação'.

Claramente as unidades de conservação estão divididas segundo uma lógica relacionada prioritariamente com critérios de proteção, na qual as unidades de proteção integral apresentam restrições ao seu uso, ocupação e exploração, mais severas do que as unidades de uso sustentável. Neste sentido, é esperado que a biodiversidade contida nas unidades de proteção integral esteja desempenhando suas funções ecológicas de forma mais acentuada do que as presentes nas unidades de uso sustentável, portanto, 
propomos o acrescimento de peso para os empreendimentos que afetem diretamente as unidades de proteção integral, conforme o descrito na tabela 5.

Tabela 5: Peso dos atributos relacionados a presença do animal atropelado em uma área de preservação.

\begin{tabular}{|l|c|}
\hline \multicolumn{1}{|c|}{ Classificação } & Peso \\
\hline \multicolumn{1}{|c|}{ Unidades de proteção integral } & 2 \\
\hline Estação Ecológica & 2 \\
\hline Reserva Biológica & 2 \\
\hline Parque Nacional & 2 \\
\hline Monumento Natural & 2 \\
\hline Refúgio de Vida Silvestre & \\
\hline \multicolumn{1}{|c|}{ Unidades de uso sustentável } & 1 \\
\hline Área de Proteção Ambiental & 1 \\
\hline Área de Relevante Interesse Ecológico & 1 \\
\hline Floresta Nacional & 1 \\
\hline Reserva Extrativista & \\
\hline Reserva de Fauna & 1 \\
\hline Reserva de Desenvolvimento Sustentável & 1 \\
\hline Reserva Particular do Patrimônio Natural & \\
\hline
\end{tabular}

Caso o empreendimento analisado não afete diretamente nenhuma unidade de conservação, o peso atribuído será 0 . O somatório do peso correspondente a cada um dos itens que compõe a severidade (densidade populacional, status de conservação, endemismo e presença em área de conservação) determinará a severidade relativa a cada uma das espécies atropeladas.

Hipoteticamente, uma espécie atropelada poderá apresentar uma determinada configuração de severidade, estando essa proposta na tabela 6. Para que se tenha a severidade do período, trecho ou da rodovia, será necessário realizar uma média aritmética do somatório dos pesos de todos os animais atropelados de forma a resultar em um único valor de severidade. Após a definição da frequência e da severidade do atropelamento, devemos correlacionar os valores, utilizando a tabela 7 para que seja possível obter o enquadramento do grau de significância de impacto ambiental.

Tabela 6: Configuração de severidade.

\begin{tabular}{|c|c|c|c|c|c|c|c|}
\hline \multirow{2}{*}{ Espécie } & \multicolumn{2}{|c|}{ Densidade populacional } & \multicolumn{2}{|c|}{ Status de conservação } & \multirow{2}{*}{ Endemismo } & \multirow{2}{*}{ Área de preservação } & Total \\
\cline { 2 - 5 } & Índice constância & peso & Categoria & Peso & & 2 & 14 \\
\hline Didelphis aurita & Acidental & 8 & LC & 2 & 2 & 2 & 14 \\
\hline
\end{tabular}

Tabela 7: Grau de significância do impacto ambiental causado pelo atropelamento.

\begin{tabular}{|c|c|c|c|c|c|c|c|c|c|}
\hline \multicolumn{8}{|c|}{ FREQUENCIA DE ATROPELAMENTO } & & \multirow{7}{*}{ 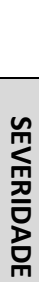 } \\
\hline \multirow[t]{7}{*}{ A } & B & C & D & $E$ & $F$ & G & H & & \\
\hline & & & & & & & & $19 \geq 21$ & \\
\hline & & & & & & & & $15 \geq 18$ & \\
\hline & & & & & & & & $12 \geq 15$ & \\
\hline & & & & & & & & $9 \geq 12$ & \\
\hline & & & & & & & & $6 \geq 9$ & \\
\hline & & & & & & & & $3 \geq 6$ & \\
\hline
\end{tabular}

Para que seja definido o enquadramento do grau de significância de impacto ambiental (baixo, médio, alto, muito alto e extremamente alto) causado pela rodovia no período estudado deverá ser utilizada a tabela 8. A tabela 8 foi denominada 'Enquadramento do grau de significância de impacto ambiental em rodovias a partir do atropelamento de vertebrados'. 
Tabela 8: Enquadramento do grau de significância de impacto ambiental em rodovias a partir do atropelamento de vertebrados.

\begin{tabular}{|l|l|}
\hline \multicolumn{1}{|c|}{ Parâmetros } & \multicolumn{1}{|c|}{ Grau de significância do impacto ambiental causado pelo atropelamento } \\
\hline & Baixo \\
\hline & Médio \\
\hline & Alto \\
\hline & Muito alto \\
\hline & Extremamente alto \\
\hline
\end{tabular}

Novamente tendo em vista o princípio da precaução, sugerimos que caso alguma espécie atropelada esteja na categoria criticamente ameaçado (ou criticamente em perigo)(CR) ou 2 - caso mais de $50 \%$ das espécies atropeladas estejam na categoria Ameaçado (ou em perigo) (EM), a classe de impacto ambiental seja considerada crítica, conforme tabela 8.

\section{CONCLUSÕES}

Através da aplicação da metodologia proposta neste trabalho, será possível analisar de uma forma inédita, os impactos ambientais causados pelo atropelamento de vertebrados em uma rodovia, certamente aportando resultados mais precisos e consistentes do que os encontrados nas análises atuais. Esperamos que a aplicação desta metodologia possibilite aos gestores de empreendimentos lineares, bem como aos órgãos fiscalizadores, uma ideia mais próxima do real impacto ambiental gerado pelo atropelamento de vertebrados.

\section{REFERÊNCIAS}

AMENT, R.; CLEVENGER, A. P.; YU, O.; HARDY, A.. An assessment of road impacts on wildlife populations in U.S. National Parks. Environmental Management, v.42, n.3, p.480-496, 2008.

BAGATINI, T.. Evolução dos índices de atropelamento de vertebrados silvestres nas rodovias do entorno da Estação Ecológica de Águas Emendadas, DF Brasil, e eficácia de medidas mitigadoras. Dissertação (Mestrado em Ecologia) Universidade de Brasília, Brasília, 2006.

BAGER, A.; FONTOURA, V.. Ecologia de Estradas no Brasil: Contexto Histórico e Perspectivas Futuras. In: BAGER, A. Ecologia de estradas: tendências e pesquisas. Lavras: EDUFLA, 2012.

BUJOCZEK, M.; CIACH, M.; YOSEF, R.. Road-kills affect avian population quality. Biological Conservation, v.144, n.3, p.1036-1039, 2011

CARDILLO, M.; MACE, G. M.; GITTLEMAN, J. L.; JONES, K. E.; BIELBY, J.; PURVIS, A.. The predictability of extinction: biological and external correlates of decline in mammals. Proceedings of the Royal Society B: Biological Sciences, v.275, p.1441-1448, 2008

CARDILLO, M.; PURVIS, A.; SECHREST, C. D. L.; GITTLEMAN, J. L.; BIELBY, J.; MACE, G. M.. Human population density and extinction risk in the world's carnivores. PLoS Biology, v.2, p.909-914, 2004
COLLEN, B.; BYKOVA, E.; STEPHEN, L.; MILNERGULLAND, E. J.; PURVIS, A.. Extinction risk: comparative analysis of Central Asian vertebrates. Biodiversity and Conservation, v.15, p.1859-1871, 2006. DOI: http://doi.org/10.1007/s10531$\underline{005-4303-6}$

DAJOZ, R.. Ecologia geral. São Paulo: Vozes, 1972.

EWERS, R. M.; DIDHAM, E. R. K.. Confounding factors in the detection of species responses to habitat fragmentation. Biological Reviews, v.81, p.117-142, 2006.

FAHRIG, L.; RYTWINSKI, T.. Effects of Roads on Animal Abundance: an Empirical Review and Synthesis. Ecology and Society, v.14, n.1, p.21, 2009.

FISHER, D. O.; BLOMBERG, S. P.; OWENS, P. F. I.. Extrinsic versus intrinsic factors in the decline and extinction of Australian marsupials. Proceedings of the Royal Society B: Biological Sciences, v.270, p.1801-1808, 2003.

FORMAN, R. T. T.; ALEXANDER, L. E.. Roads and their major ecological effects. Annual Review of Ecology and Systematics, v.29, p.207-231, 1998.

FRITZ, S. A.; BININDA-EMONDS, O. R. P; PURVIS, A. Geographical variation in predictors of mammalian extinction risk: big is bad, but only in the tropics. Ecology Letters, v.12, p.538-549, 2009.

HUCK, M.; JĘDRZEJEWSKI, W.; BOROWIK, T.; MIŁOSZCIELMA, M.; SCHMIDT, K, J. Ę.; DRZEJEWSKA, B.; NOWAK, S. 
MYS, Ł.; AJEK, R. W.. Habitat suitability, corridors and dispersal barriers for large carnivores in Poland. ActaTheriologica, v.55, n.2, p.177-192, 2010. DOI: http://doi.org/10.4098/i.at.0001-7051.114.2009

JACKSON, N. D.; FAHRIG, L.. Relative effects of road mortality and decreased connectivity on population genetic diversity. Biological Conservation, v.144, p.3143-3148, 2011.

JOHNSON, C. N.. Determinants of loss of mammal species during the Late Quaternary 'megafauna' extinctions: life history and ecology, but not body size. Proceedings of the Royal Society B: Biological Sciences, v.269, p.2221-2227, 2002.

JONES, K. E.; PURVIS, A.; GITTLEMAN, J. L.. Biological correlates of extinction risk in bats. The American Naturalist, v.161, p.601-614, 2003.

LAURANCE, W. F.; CLEMENTS, G. R.; SLOAN, S.; O'CONNELL, C. S.; MUELLER, N. D.; GOOSMEN, M.; VENTER, O.; EDWARDS, D. P.; PHALAN, B.; BALMFORD, A.; REE, R.; ARREA, I. B.. A global strategy for road building. Nature, v.513, p.229-232, 2014.

MACE, G. M.; COLLAR, N. J.; GASTON, K. J.; HILTON-TAYLOR, C.; AKCAKAYA, H. R.; LEADER-WILLIAMS, N.; MILNERGULLAND, E. J.; STUART, S. N.. Quantification of extinction risk: IUCN's system for classifying threatened species. Conservation Biology, v.22, p.1424-1442, 2008.
PARKS, S. A.; HARCOURT, A. H.. Reserve Size, Local Human Density, and Mammalian Extinctions in U.S. Protected Areas. Conservation Biology, v.16, n.3, p.800-808, 2002.

PRADA, C. S.. Atropelamento de vertebrados silvestres em uma região fragmentada do nordeste do Estado de São

Paulo: quantificação do impacto e análise de fatores envolvidos. Dissertação (Mestrado em Ecologia e Recursos Naturais) - Universidade Federal de São Carlos, São Paulo, 2004.

PURVIS, A.; GITTLEMAN, J. L.; COWLISHAW, G.; MACE, G.. Predicting extinction risk in declining species. Proceedings of the Royal Society B: Biological Sciences, v.267, p.1947-1952, 2000.

REIS, P. L.. Estudos de Impactos Ambientais nas rodovias do Estado de São Paulo: uma revisão crítica. Monografia (Graduação em Ciências Biológicas) - Universidade Estadual Paulista 'Júlio de Mesquita Filho', Botucatu, 2015.

ROCHA, C. D. F.; BERGALLO, H. G.; ALVES, M. A. S.; SLUYS, M.; MAZZONI, R.; SANTOS, S. B.. Fauna de ambientes interiores. In: BASTOS, M.; CALLADO, C. H.. O ambiente da Ilha Grande. Rio de Janeiro: UERJ, 2009.

VIÉ, J. C.; HILTON-TAYLOR, C.; STUART, S. N.. Wildlife in a changing world: an analysis of the 2008. Gland: IUCN, 2009.

A CBPC - Companhia Brasileira de Produção Científica (CNPJ: 11.221.422/0001-03) detém os direitos materiais desta publicação. Os direitos referem-se à publicação do trabalho em qualquer parte do mundo, incluindo os direitos às renovações, expansões e disseminações da contribuição, bem como outros direitos subsidiários. Todos os trabalhos publicados eletronicamente poderão posteriormente ser publicados em coletâneas impressas sob coordenação da Sustenere Publishing, da Companhia Brasileira de Produção Científica e seus parceiros autorizados. Os (as) autores (as) preservam os direitos autorais, mas não têm permissão para a publicação da contribuição em outro meio, impresso ou digital, em português ou em tradução. 